Journal of Patient-Centered

\title{
What Are the Recommended Timing and Screening Modalities for Women at Higher Risk of Developing Breast Cancer? A Clin-IQ
}

Summer Jatala

Shawn Fitzgerald

Pamela Tietze

Kalyanakrishnan Ramakrisnan

Laine H. McCarthy

Elizabeth Wickersham

Follow this and additional works at: https://aah.org/jpcrr

Part of the Diagnosis Commons, Neoplasms Commons, Oncology Commons, Public Health Education and Promotion Commons, and the Women's Health Commons

\section{Recommended Citation}

Jatala S, Fitzgerald S, Tietze P, Ramakrisnan K, McCarthy LH, Wickersham E. What are the recommended timing and screening modalities for women at higher risk of developing breast cancer? A Clin-IQ. J Patient Cent Res Rev. 2015;2:38-42. doi: 10.17294/2330-0698.1051

Published quarterly by Midwest-based health system Advocate Aurora Health and indexed in PubMed Central, the Journal of Patient-Centered Research and Reviews (JPCRR) is an open access, peer-reviewed medical journal focused on disseminating scholarly works devoted to improving patient-centered care practices, health outcomes, and the patient experience. 


\title{
What Are the Recommended Timing and Screening Modalities for Women at Higher Risk of Developing Breast Cancer? A Clin-IQ
}

Summer Jatala, MD, Shawn Fitzgerald, DO, Pamela Tietze, MD, Kalyanakrishnan Ramakrishnan, MD, Laine H. McCarthy, MLIS, Elizabeth Wickersham, MD

Family Medicine Residency Program, Department of Family and Preventive Medicine, University of Oklahoma Health Sciences Center, Oklahoma City, OK

\begin{abstract}
Early detection of breast cancer is desirable to prevent progression to advanced disease. This subject has been one of significant study and debate for women at normal risk, and recommendations continue to evolve. However, with regard to women at high risk, the recommendations from various health care professional organizations, including the U.S. Preventive Services Task Force, are different and also inconsistent concerning when to begin screening and which modalities should be used. We review several randomized controlled trials and consensus opinions regarding when to begin screening for breast cancer and how to best screen women at high risk. Specifically, we address women with known personal history of breast cancer, prior mantle radiation or specific family history (including genetic family history) of breast cancer. The purpose of this inquiry is to present current evidence and suggest a clinical pathway regarding the screening of women at high risk for breast cancer. ( $\mathrm{J}$ Patient-Centered Res Rev. 2015;2:38-42.)
\end{abstract}

Keywords breast cancer, screening for breast cancer, mammography, magnetic resonance imaging

\section{Clinical Question}

In women with increased risk factors for breast cancer, at what age should screening begin and with what modality?

\section{Answer}

Women with a family history of breast cancer should have annual digital mammography, as warranted, beginning at age 30 or at 10 years younger than when the youngest family member was diagnosed with breast cancer (but not before age 25). Contrastenhanced magnetic resonance imaging (CE-MRI) with digital mammography as well as annual clinical breast examination beginning at age 30 is recommended in screening women with known genetic mutations enhancing breast cancer risk or who have first-degree relatives with these mutations. Women treated with

Correspondence: Elizabeth Wickersham, MD, Department of Family \& Preventive Medicine, University of Oklahoma Health Sciences Center, 900 NE 10th Street, Oklahoma City, Oklahoma, 73104, Email: elizabeth-wickersham@ouhsc.edu mantle radiation should undergo annual CE-MRI, digital mammography screening and clinical breast exam commencing 8 years after completion of treatment, or at age 25 .

Date answer was determined: October 2014

\section{Level of evidence: A}

Inclusion criteria: Published systematic reviews, meta-analyses, cohort studies and consensus guidelines on the timing of and modalities used to screen for breast cancer in women with a history of breast cancer in a first-degree relative.

Exclusion criteria: Non-English language articles.

\section{SUMMARY OF THE ISSUES}

Breast cancer is the most common cancer among women (12\% lifetime risk) and the principal cause of cancer-related death for more than $3 \%$ of women. ${ }^{1}$ According to radiology society recommendations, earlier detection through imaging in low-risk patients has reduced breast cancer-related mortality by about 
one-third, ${ }^{1}$ although this type of early screening is a hotly debated topic. The U.S. Preventive Services Task Force currently recommends biennial screening mammography in average-risk women 50-74 years of age; for women less than 50 years old, the recommendation is for personal risk-based screening. ${ }^{2}$ This is a controversial change from prior recommendations of annual screening for women, with much of the debate coming from the American Cancer Society, American College of Radiology, and American College of Obstetrics and Gynecology groups that currently recommend annual mammography screening starting at age 40 .

Adding to this controversy is the most recent study released by the Canadian National Breast Screening Study in February 2014, which concluded that annual screening of low-risk women 40-59 years of age does not reduce breast cancer beyond that of physical examination or usual care. ${ }^{3}$ This study was highly criticized by the American College of Radiology and the Society of Breast Imaging. These organizations called the study "highly flawed" and referenced outdated equipment use and poor methodology. However, the study's authors refuted these assertions and stood by their study. ${ }^{4}$

Mammography in women under age 50 is complicated by dense breast tissue that leads to a higher rate of false-positive breast cancer diagnoses, anxiety generated by the positive screenings, and risk of complications engendered by invasive procedures, thus the controversy surrounding routine mammography in this age group. Metaanalyses have reported a $20 \%$ reduction in 15 -year mortality from breast cancer for women in their 40s. ${ }^{5}$ Put another way, the same authors estimated that " 2 out of 1,000 women who regularly undergo mammography during their 40s might owe their lives to mammography." 6

In addition to the numerous and inconsistent recommendations for screening in the low-risk population, there remains increased confusion regarding concise recommendations for screening the high-risk patient population, and the availability of different screening modalities and conflicting recommendations regarding timing of screening has added to this confusion. ${ }^{2}$ Data from several cohort studies, a systematic review of randomized control trials and consensus opinions all recommend that women with specific risk factors placing them at higher risk than the general population be screened beginning at or after age 30 , or at 10 years from the age of diagnosis of breast cancer in the youngest relative (but not before age 25). ${ }^{1,7-9}$ Risk factors to identify include a positive personal history of breast cancer (excluded with some protocols $^{7}$ but not others ${ }^{1}$ ), family history of breast cancer in a first- or second-degree relative, personal or family history of specific genetic mutations, personal or family history of ovarian cancer in a first- or seconddegree relative, and prior mantle radiation. ${ }^{7}$

Multiple factors can affect the predictive value of screening tests, including age, screening interval, equipment quality/age, breast tissue density and proficiency of the radiologist. Digital mammography has generally become the standard of care in breast cancer screening. Contrast-enhanced magnetic resonance imaging (CE-MRI) is advocated in screening high-risk women, particularly in younger women or those with dense breast tissue, ${ }^{1,8,10}$ although the expense, increased risk of false positives and lack of universal availability limit its usefulness. ${ }^{4}$

\section{SUMMARY OF THE EVIDENCE}

A British prospective multicenter cohort study compared CE-MRI and digital mammography in highrisk younger women. This population was chosen because of the dense breast tissue present and known decreased sensitivity of mammography in this age group. The inclusion criteria for the study included women 35-49 years of age with a high probability of BRCA1, BRCA2 or TP53 genetic mutations or a strong family history of breast cancer. ${ }^{8}$ CE-MRI yielded a higher tumor detection rate than mammography alone $(\mathrm{P}=0.01)$. CE-MRI had a sensitivity of $77 \%$ (confidence interval [CI]: 60-90) and a specificity of $81 \%$ (CI: $80-83$ ) compared with mammography sensitivity of $40 \%$ (CI: 24-58) and specificity of $93 \%$ (CI: 92-95). Combined imaging modalities (CE-MRI and/or mammography) had a sensitivity of 94\% (CI: 81-99) and specificity of 77\% (CI: 75-79). CE-MRI detected more breast cancers than mammography (92\% vs. $23 \%)$ in women specifically with $B R C A 1$ genetic mutations $(\mathrm{P}=0.004) .{ }^{11}$ 
To determine if earlier breast cancer screening was beneficial in women with more risk factors for the disease, a more recent British study screened a cohort 7,475 women $35-50$ years of age with enhanced risk of developing early breast cancer due to a strong family history (a first-degree relative $<40$ years of age or a first- or second-degree relative $<60$ years of age with breast cancer). ${ }^{10}$ In addition to family history data, other risk factor information was collected, including age at menarche/menopause, parity, use of female hormones and age at first birth. Women who were found to have a $>16.7 \%$ risk of developing breast cancer in their lifetime were selected for annual surveillance until age 50 .
Women with a lifetime risk of $>25 \%$ were offered 18 monthly screenings in addition to their three yearly screenings from age 51 to 60 , which is normally covered by the National Health Service Breast Screening Program. Study participants were initially screened with single-view mammography until 1999 and two-view mammography thereafter. Clinical breast examination was performed on all women by specialized nursing staff before mammography, and breast self-examination instruction was offered to all women. Participants were encouraged to return if they detected any worrisome changes or findings on breast self-exam. Earlier screening resulted in the detection of 165 breast cancers, 106 of which were in

\section{Known Family History of Breast Cancer?}

Yes $\rightarrow$ Annual screening mammography and clinical breast examination (CBE) at age 30 or 10 years earlier than the age of diagnosis of the youngest family member with breast cancer (but not before age 25). ${ }^{1}$

No $\rightarrow$ Biennial screening mammography age 50-74 per U.S. Preventive Services Task Force recommendations unless indicated earlier by breast pain or mass, skin changes, nipple discharge or axillary lymph node enlargement. ${ }^{2}$

\section{Personal History of Breast or Ovarian Cancer?}

Yes $\rightarrow$ Annual contrast-enhanced magnetic resonance imaging (CE-MRI) + digital mammography and CBE beginning the year after diagnosis of breast or ovarian cancer. ${ }^{1}$

$N o \rightarrow$ Biennial screening mammography with CBE unless clinically indicated as above. ${ }^{2}$

\section{Presence of BRCA1, BRCA2 or TP53 Genetic Mutations, and/or Known Family History of Ovarian Cancer?}

Yes $\rightarrow$ Annual CE-MRI + digital mammography and CBE starting at age 30 or 10 years earlier than the age of diagnosis of the youngest family member with breast cancer (but not before age 25). ${ }^{8}$

No $\rightarrow$ Biennial screening mammography with CBE unless clinically indicated as above. ${ }^{2}$

\section{Personal History of Mantle Radiation?}

Yes $\rightarrow$ Annual CE-MRI + digital mammography and CBE 8 years after completion of mantle radiation treatment. ${ }^{1}$

No $\rightarrow$ Biennial screening mammography with CBE unless clinically indicated as above. ${ }^{2}$

Figure 1. Breast cancer screening questions and clinical pathway. 
women $<50$ years of age. The cancers detected were of more advanced grade, with a tendency for younger women to have grade 3 tumors $(\mathrm{P}=0.08) .{ }^{9}$

The Society of Breast Imaging and American College of Radiology collaboratively appraised multiple peerreviewed published scientific data from meta-analyses of European and North American randomized controlled trials, expert consensus opinions and supporting guidelines to provide direction on both the type of imaging to use and when to start screening women with specific risk factors for breast cancer. Identified specific risk factors supporting screening before age 40 include women with genetic mutations known to increase breast cancer risk by age 40 and women who underwent mantle field radiation at $10-30$ years of age. ${ }^{1}$

A cross-sectional study comparing screening mammography in women with history of breast cancer in one or more first-degree relatives against those of similar age without such history found more cancers in the first group $(6.1 / 1,000$ vs. 4.0/1,000), corresponding to that in average-risk women a decade older. However, the sensitivity of mammography increases with age (87.0\% for age 60-69 years; $67.9 \%$ for age $30-39$ ) and is less beneficial in younger women. Nevertheless, mammography has a higher positive predictive value $(3.7 \%$ in the first group vs. $2.9 \%, \mathrm{P}=0.001)$ in those with a family history positive for breast cancer. ${ }^{12}$

A suggested clinical pathway for screening women at high risk for breast cancer is illustrated in Figure 1.

\section{CONCLUSIONS}

For women with specific personal or family risk factors placing them at higher risk for developing breast cancer versus the general population, breast cancer screening beginning prior to age 50 with digital mammography or, in specific circumstances, digital mammography combined with contrast-enhanced magnetic resonance imaging is recommended. Earlier screening (beginning at 30 years) appears warranted in this high-risk population even though mammography is less sensitive in younger women. ${ }^{12} \mathrm{CE}-\mathrm{MRI}$ is more sensitive in detecting malignancy in women with known personal genetic mutations for breast or ovarian cancer, and/or with a prior personal history of mantle radiation treatment. Digital mammography combined with CE-MRI should be offered to these women. As part of the process of recommending earlier breast cancer screening, a discussion of the risks and benefits of earlier screening should be performed with these high-risk women.

\section{Patient-Friendly Recap}

- Women considered at high risk for breast cancer include those who have a personal or family history of the disease, those who have breast cancer-related genes or those who have been exposed to radiation.

- Various recommendations for how and when to screen these high-risk women for breast cancer are inconsistent.

- The authors provide an algorithm for screening patients at high risk for breast cancer in Figure 1.

\section{Acknowledgments}

Clin-IQ (Clinical Inquiries) is a required resident/ faculty scholarly activity that takes questions from community practitioners and feeds the answers back out to improve patient care. Ms. McCarthy received support from Oklahoma Shared Clinical \& Translational Resources, funded by grant NIGMS U54GM104938, NIGMS/NIH.

\section{Conflicts of Interest}

None.

\section{REFERENCES}

1. Lee CH, Dershaw DD, Kopans D, et al. Breast cancer screening with imaging: recommendations from the Society of Breast Imaging and the ACR on the use of mammography, breast MRI, breast ultrasound, and other technologies for the detection of clinically occult breast cancer. $J$ Am Coll Radiol. 2010;7:18-27.

2. U.S. Preventive Services Task Force. Screening for breast cancer: U.S. Preventive Services Task Force recommendation statement. Ann Intern Med. 2009;151:716-26.

3. Miller AB, Wall C, Baines CJ, Sun P, To T, Narod SA. Twenty five year follow-up for breast cancer incidence and mortality of the Canadian National Breast Screening Study: randomised screening trial. BMJ. 2014;348:g366.

4. Printz C. Mammogram debate flares up: Latest breast cancer screening study fuels controversy. Cancer. 2014;120:1755-6. 
5. Fletcher SW, Elmore JG. Clinical practice. Mammographic screening for breast cancer. $N$ Engl J Med. 2003;348:1672-80.

6. Fletcher SW, Elmore JG. Authors' reply to letters to the editor regarding "Mammographic Screening for Breast Cancer." N Engl J Med. 2003;349:612.

7. Kaiser Permanente Care Management Institute. Breast cancer screening clinical practice guideline. Oakland, CA: Kaiser Permanente Care Management Institute, 2010.

8. Saslow D, Boetes C, Burke W, et al. American Cancer Society guidelines for breast screening with MRI as an adjunct to mammography. CA Cancer J Clin. 2007;57:75-89.

9. Maurice A, Evans DG, Affen J, Greenhalgh R, Duffy SW, Howell A. Surveillance of women at increased risk of breast cancer using mammography and clinical breast examination: further evidence of benefit. Int J Cancer. 2012;131:417-25.
10. Ahern CH, Shih YC, Dong W, Parmigiani G, Shen Y. Costeffectiveness of alternative strategies for integrating MRI into breast cancer screening for women at high risk. Br J Cancer. 2014;111:1542-51.

11. Leach MO, Boggis CR, Dixon AK, et al. Screening with magnetic resonance imaging and mammography of a UK population at high familial risk of breast cancer: a prospective multicentre cohort study (MARIBS). Lancet. 2005;365: 1769-78.

12. Kerlikowske K, Carney PA, Geller B, et al. Performance of screening mammography among women with and without a first-degree relative with breast cancer. Ann Intern Med. 2000;133:855-63.

(C) 2015 Aurora Health Care, Inc. 